\title{
IMPACT DES FLUCTUATIONS GLACIAIRES ET DES ANCIENNES ACTIVITÉS MINIÈRES D'ALTITUDE SUR LA SÉDIMENTATION LACUSTRE PROGLACIAIRE AU COURS DU TARDIGLACIAIRE ET DE L'HOLOCÈNE DANS LE MASSIF DES GRANDES ROUSSES
}

\author{
ALPES OCCIDENTALES, FRANCE
}

\author{
IMPACT OF GLACIER FLUCTUATIONS AND HIGH ALTITUDE MINING ACTIVITIES ON LATE GLACIAL- \\ Holocene PROGLACIAL LACUSTRINE SEDIMENTATION IN THE GRANDES RoUSSES MASSIF \\ WESTERN ALPS, FRANCE
}

\begin{abstract}
Emmanuel CHAPRON ${ }^{1,2}$, Marie-Christine BAILLY-MAITRE ${ }^{3}$, Flavio ANSELMETTI ${ }^{1,4}$, Hervé GUYARD ${ }^{5}$, Gulllaume SAINT ONGE ${ }^{5}$, Marc DESMET ${ }^{6,12}$, CATHerine CHAUVEL ${ }^{7}$, ThiERry WINIARSKI ${ }^{6}$, OLIVIER MAGAND ${ }^{8}$, FABIEN ARNAUD $^{6}$, LAURENT CHARLET ${ }^{9}$, Philip DELiNE ${ }^{6}$, Michel MAGNY ${ }^{10}$, Marie-AntoinetTe MÉliÈres ${ }^{8,11}$.
\end{abstract}

\author{
${ }^{1}$ Geological Institute, ETH Zurich, Suisse \\ ${ }^{2}$ Institut des Sciences de la Terre d'Orléans, UMR 6113 CNRS-Université d'Orléans, Orléans, France \\ ${ }^{3}$ Laboratoire d'Archéologie Médiévale Méditerranéenne, UMR 6572 CNRS-Université de Provence, Aix en Provence, France \\ ${ }^{4}$ Sedimentology Group, Department of Surface Waters, EAWAG, Dübendorf, Suisse \\ ${ }^{5}$ ISMER, Université de Rimouski, Rimouski, Canada \\ ${ }^{6}$ Laboratoire EDYTEM/CNRS, Université de Savoie, Campus scientifique, F 73376 Le Bourget du Lac cedex \\ ${ }^{7}$ Laboratoire de Géodynamique des Chaines Alpines, UMR 5025 CNRS-Observatoire de Grenoble, St Martin d'Hères, France \\ ${ }^{8}$ Laboratoire de Glaciologie et de Géophysique de l'Environnement, UMR 5183 CNRS-Observatoire de Grenoble, St Martin d'Hères, France \\ ${ }^{9}$ Laboratoire de Géophysique Interne et Tectonophysique, Observatoire de Grenoble, St Martin d'Hères, France \\ ${ }^{10}$ Laboratoire de Chrono Environnement, UMR 6249 CNRS-Université de Franche Comté, Besançon, France \\ ${ }^{11}$ Institut de la Montagne, Université de Savoie, campus scientifique, F 73376 Le Bourget du Lac cedex \\ ${ }^{12}$ INRP \& ENTPE - Université de Lyon \\ Contact : emmanuel.chapron@univ-orleans.fr
}

\begin{abstract}
RÉSUMÉ
Des datations à la base de carottages localisés à partir de profils « sismique réflexion » à plus de $2500 \mathrm{~m}$ d'altitude dans les remplissages des lacs proglaciaires de Bramant et de Blanc Huez (Massif des Grandes Rousses) indiquent que ces deux lacs ont été déglacés depuis la fin du Tardiglaciaire et que leurs bassins versants ont été très faiblement englacés durant la première partie de l'Holocène. Le développement d'un néoglaciaire impliquant le retour d'une sédimentation détritique dans ces lacs proglaciaires est ensuite marqué par des fluctuations rapides de l'activité glaciaire. Durant les périodes du Bronze ancien et récent en particulier, la prédominance d'une sédimentation organique dans le Lac de Bramant implique que les glaciers étaient probablement plus réduits qu'aujourd 'hui dans le nord du massif. Dans l'ensemble du massif, l'extension maximale des glaciers holocènes semble avoir été atteinte au cours du dernier millénaire durant le Petit Age Glaciaire. La synthèse de fouilles archéologiques d'altitude dans ce massif et l'identification dans les sédiments lacustres de pics de métaux permet de préciser l'évolution de la paléo-métallurgie en altitude. Un pic de Cuivre entre 3770 et 3870 cal BP dans le Lac de Bramant peut ainsi être lié à la découverte de sites d'exploitation d'un filon de chalcopyrite entre 2200 et $2600 \mathrm{~m}$ d'altitude à proximité du bassin versant. Au sud du massif, deux pics d'Argent reconnus dans les sédiments récents du Lac Blanc Huez peuvent également être reliés au développement d'une mine de Plomb argentifère en bordure du lac entre AD 1236 et 1330. Par contre, l'origine d'un pic d'Argent plus ancien dans le Lac Blanc Huez et celle d'un pic de Plomb et de Cuivre durant l'apogée de la période romaine entre AD 115-330 dans le lac de Bramant restent encore à expliquer.
\end{abstract}

Mots-CLÉS : LACS PROGLACIAIRES, MASSIF DES GRANDES ROUSSES, FLUCTUATIONS GLACIAIRES, PALÉO MÉTALLURGIE, PALÉO ENVIRONNEMENTS. 


\begin{abstract}
Radiocarbon dating at the base of sediment cores retrieved from high altitude proglacial lakes Bramant and Blanc Huez (Grandes Rousses Massif, $2500 \mathrm{~m}$ altitude) based on high resolution seismic reflection profiling, suggest that these lakes were ice free since the end of the Lateglacial period, and that their catchment areas were largely free of glaciers during the first part of the Holocene. The development of a neoglacial period implying increasing clastic sedimentation in these proglacial lakes is then characterized by several rapid glacier fluctuations. During the Early and Late Bronze Age periods, for example, enhanced organic sedimentation in Lake Bramant suggest that glaciers in this part of the massif were probably smaller than today. The maximum extension of Holocene glaciers in this massif was then reached during the Little Ice Age. A synthesis of archaeological investigations and the identification of several metallic contaminations in lacustrine sediments, allow documenting the evolution at high altitude of mining activities. High content of Copper between 3770-3870 cal BP in Lake Bramant sediments can for example be related to Early Bronze Age mining artefacts recently identified between 2200 and $2600 \mathrm{~m}$ within chalcopyrite ore deposits next to the catchment area of Lake Bramant in the Northern part of the massif. In the southern part of the massif, two peaks highlighting high content of Silver in Lake Blanc Huez recent sediments can similarly be related to a well documented Lead Silver mining industry at the lake shore between AD 1236 and 1330. The origins of an older peak in Silver in Lake Blanc Huez and of a clear peak in Copper and Lead during the Roman period between AD 115-330 in Lake Bramant, are however not clearly understood.
\end{abstract}

KEYWORDS: PROGLACIAL LAKES, GRANDES ROUSSES MASSIF, GLACIER FLUCTUATIONS, PALEO METALLURGY, PALEO ENVIRONMENTS.

\title{
INTRODUCTION
}

Dans le contexte du réchauffement climatique en cours et de son impact sur les ressources en eaux ou sur les sociétés, les recherches archéologiques et paléo-environnementales menées en milieu montagnard sont marquées par un indéniable regain d'intérêt. Dans le cadre des programmes de recherche pluridisciplinaire, telles que le GDR Juralp du CNRS, le PCR « Evolution des activités minières dans le Massif des Grandes Rousses » et le programme de recherche de l'Institut de la Montagne « CLIMALP : Archives passées Climat/ Environnement dans les Alpes Nord-Occidentales ", un effort particulier est ainsi orienté depuis plusieurs années sur les reconstitutions paléo-environnementales (variations des niveaux lacustres, des bilans glaciaires, de la végétation) et leurs relations éventuelles avec les chronologies ainsi que les modalités du peuplement et de l'exploitation des écosystèmes de moyenne et de haute montagne dans les Alpes françaises (développement de l'agriculture, du déboisement, de la métallurgie et, plus récemment, de l'hydroélectricité).

De nombreuses études couplant l'archéologie et la géochimie de sédiments holocènes prélevés en France au sein d'estuaires (Alfonso et al., 2001), de tourbières de moyenne altitude (Galop et al., 2001 ; Monna et al., 2004 ; Jouffroy-Bapicot et al., 2007) et de sédiments lacus- tres d'altitude (Arnaud et al., 2005) ont démontré que des retombées atmosphériques anciennes de métaux associées à l'exploitation locale ou régionale de ressources minérales, peuvent être clairement identifiées et datées. Ces travaux soulignent cependant qu'il est préférable pour cela d'étudier des séquences présentant des taux de sédimentation relativement importants, de préciser en parallèle l'évolution des activités anthropiques à partir d'analyses palynologiques, mais également d'établir la signature du bruit de fond géochimique de différents éléments au sein des séquences sédimentaires étudiées. Ces travaux nous permettent ainsi aujourd'hui de mieux comprendre l'évolution des activités métallurgiques anciennes dans les Pyrénées,

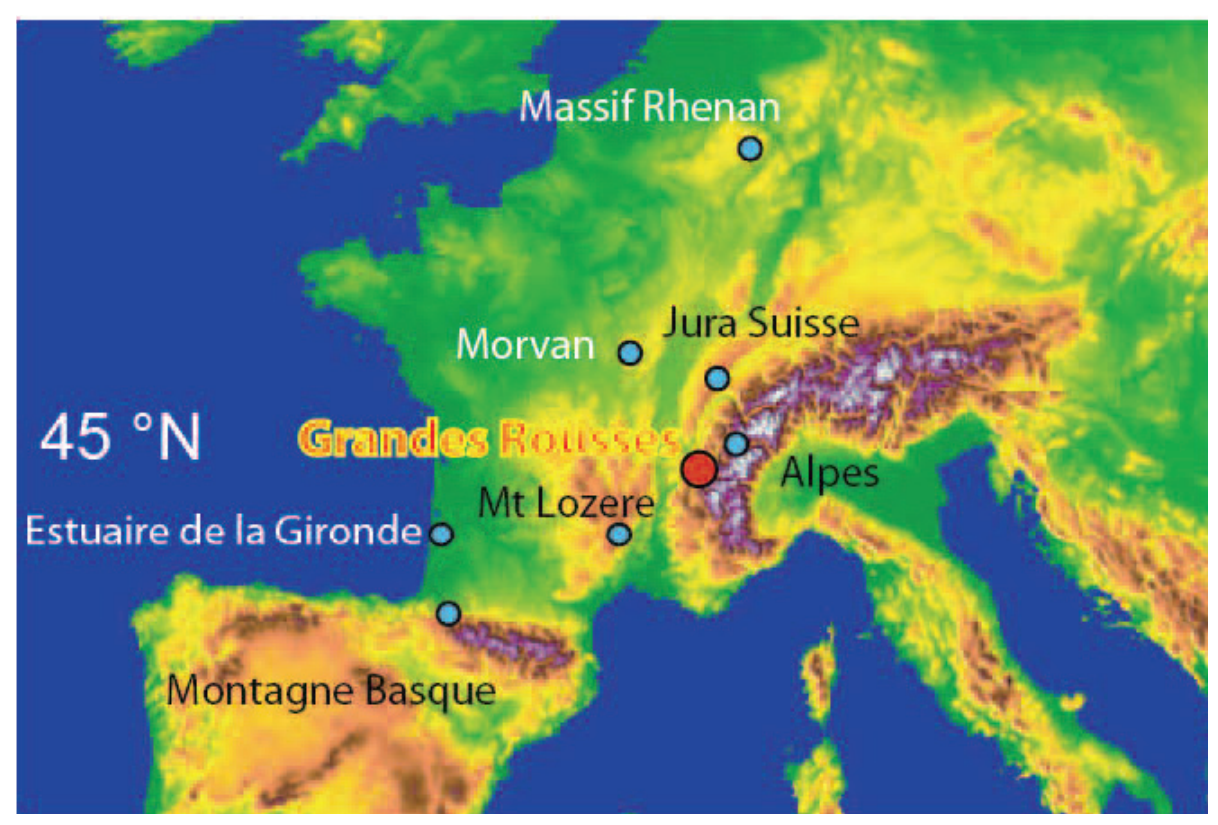

Figure 1 - Localisation du Massif des Grandes Rousses dans les Alpes Occidentales Françaises (point rouge) et des principales archives sédimentaires holocènes ayant enregistrées des pics de pollutions atmosphériques liées au développement des activités métallurgiques anciennes en France et à proximité de ses frontières avec la Suisse et l'Allemagne. 
le Massif Central et les Alpes (Figure 1). Les premières activités métallurgiques identifiées remonteraient ainsi à l'Age du Bronze moyen (1700 ans BC) dans le pays Basque (Monna et al., 2004). Une exploitation importante du Plomb semble également se généraliser durant la période Romaine et être associée à des teneurs fortes en plomb dans les remplissages sédimentaires jusqu'à plus de $2000 \mathrm{~m}$ d'altitude dans les Alpes (Arnaud et al., 2005). L'établissement de la signature isotopique de retombées atmosphériques sédimentées dans des archives naturelles en Europe et celle des minerais collectés sur sites archéologiques, suggèrent également que durant l'Empire Romain, plusieurs sites d'exploitation du Plomb ont pu exister en Europe Occidentale (Rosman et al., 1997 ; Boutron et al., 2004 ; DuraliMueller et al., 2007).

La ligne d'équilibre des glaciers dans les Alpes françaises Occidentales est aujourd'hui située aux environs de 2900 m d'altitude (Vincent, 2002), mais elle a fluctué de façon remarquable notamment au cours du dernier millénaire, en réponse aux changements climatiques durant le Petit Age Glaciaire (PAG, AD 1350-1880 ; Vincent et al., 2005 ; Chapron et al., 2002 ; 2007). A la fin de la déglaciation (Dorthe-Monachon, 1988), un retrait généralisé des glaciers dans les Alpes semble avoir culminé durant l'optimum climatique qui a caractérisé la fin de l'Holocène ancien et la majorité de l'Holocène moyen (Leeman et Niessen, 1994; Hormes et al., 2001). Durant cette période, les Alpes auraient ainsi été nettement moins englacées qu'aujourd'hui, jusqu'au développement d'une période néoglaciaire dès 5300 ans cal BP environ (Magny et Haas, 2004). La généralisation de ce néoglaciaire semble cependant avoir été relativement asynchrone dans les différents massifs Européens (Nesje et al., 2000 ; Deline et Orombelli, 2005 ; Solomina et al., 2007). Au cours des derniers 3500 ans cal BP (c.a.d. depuis l'Age du Bronze moyen), d'importantes fluctuations glaciaires ont également pu être identifiées dans les massifs alpins (Holzhauser et al., 2005 ; Deline et Orombelli, 2005).

Dans un tel contexte, afin de préciser l'évolution des activités humaines dans les Alpes au-delà de $2000 \mathrm{~m}$ d'altitude au cours de l'Holocène, il semble donc nécessaire de préciser dans le détail l'évolution des fluctuations glaciaires dès la fin du Tardiglaciaire et de documenter d'éventuelles pollutions atmosphériques liées au développement de la paléo-métallurgie en altitude.

Les objectifs de ce travail sont donc ici :

- de présenter brièvement la méthodologie mise en œuvre dans le Massif des Grandes Rousses $\left(45^{\circ} \mathrm{N}\right.$, Alpes Occidentales françaises) combinant différents types de datations et l'intégration de recherches archéologiques, géomorphologiques et sédimentologiques réalisées à plus de $2500 \mathrm{~m}$ d'altitude

- et d'établir une première synthèse de l'état de nos connaissances sur l'évolution des fronts glaciaires depuis la fin du Tardiglaciaire et des activités minières d'altitude depuis l'Age du Bronze.

\section{I - ConTEXTE GÉNÉRAL}

Le Massif des Grandes Rousses situé à $45^{\circ} 7^{\prime} \mathrm{N}$ et $6^{\circ} 6^{\prime} \mathrm{E}$ se caractérise aujourd'hui par des plateaux et des cirques glaciaires développés entre 2500 et $3400 \mathrm{~m}$ d'altitude (Figure 2). Deux glaciers situés au Sud (Sarennes) et au Nord (St Sorlin) du massif sont respectivement instrumentés depuis 1949 et 1957 et sont considérés comme représentatifs pour les Alpes Nord Occidentales (Torinesi et al., 2002 ; Vincent, 2002). Cinq générations de cordons morainiques ont été également cartographiées dans ce massif (Flusin et al., 1909 ; Monjuvent et Chardon, 1989) entre 1700 et $2600 \mathrm{~m}$ d'altitude. Le cordon situé à $2600 \mathrm{~m}$ corrélé à la dernière avance des glaciers durant le PAG entre AD 1820-1850, se situe notamment en amont des lacs proglaciaires aux environs de $2500 \mathrm{~m}$ d'altitude au Sud (Lac Blanc Huez) et au Nord (Lac Bramant) du massif (Edouard, 1994 ; Chapron et al., 2007 ; Guyard et al., 2007). Ces deux lacs sont respectivement associés à deux générations de cordons morainiques : le cordon du lac Blanc au Sud et le cordon du Lac Bramant au Nord du massif (Figure 2). Au niveau de la station de ski de l'Alpe d'Huez, les tourbes coiffant deux cordons morainiques développés à 1600 et $1700 \mathrm{~m}$ d'altitude, ont été datées d'âge Tardiglaciaire (Dryas ancien) par palynologie (Chardon, 1991). Les lacs Blanc Huez et Bramant ont donc probablement été déglacés durant la transition Tardiglaciaire-Holocène et contiennent potentiellement un enregistrement continu des changements environnementaux de l'Holocène (Chapron et al., 2007 ; Guyard et al., 2007).

L'érosion glaciaire dans ce massif composé essentiellement de gneiss, schistes et roches sédimentaires triasiques a été favorisée par le développement d'une intense fracturation d'orientation NS et NW-SE lors de la formation du massif. Des prospections archéologiques menées depuis 1985 en Oisans ont permis d'identifier plusieurs ensembles miniers anciens dans le massif des Grandes Rousses (Bailly-Maitre et BrunoDupraz, 1994) dont le site du village minier de Brandes (XII-XIV siècles) à $1830 \mathrm{~m}$ au Sud du Lac Blanc Huez (Figure 2). A proximité de ce village, une exploitation de plomb argentifère dans des gangues de baryte s'est 

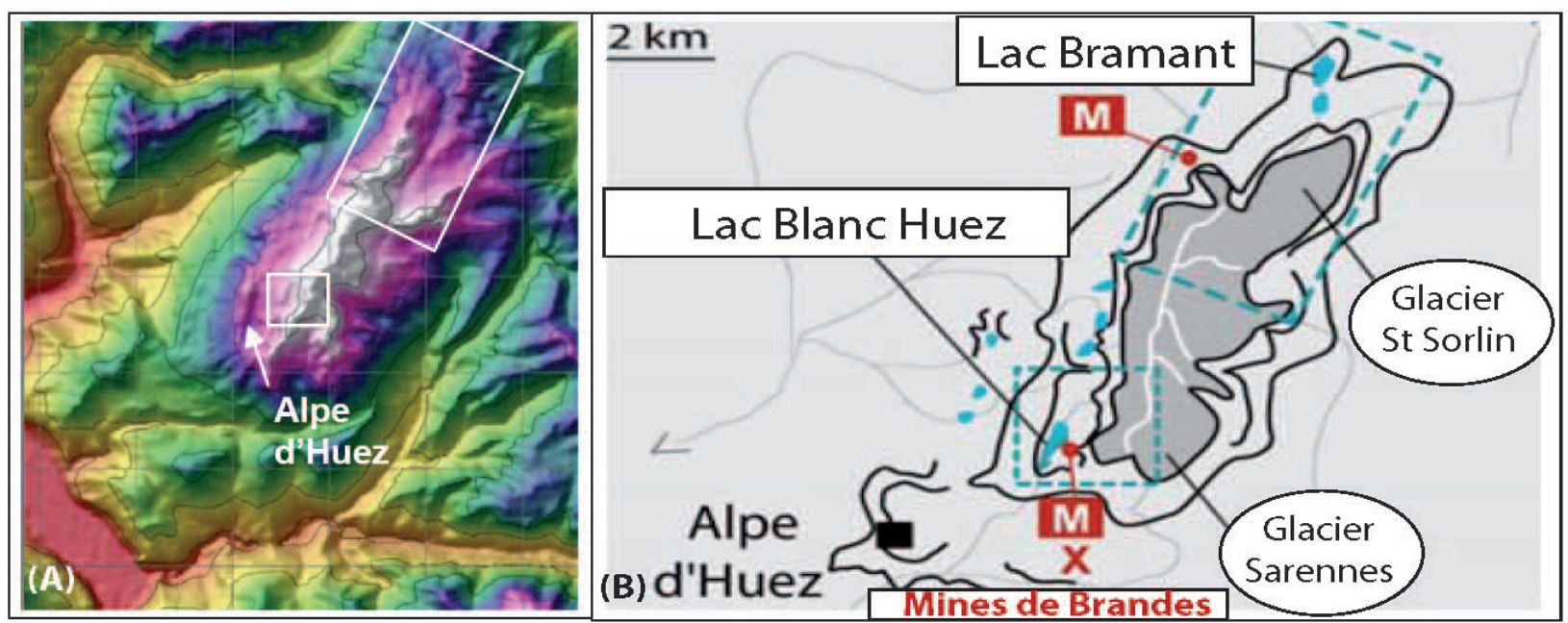

Figure 2 - (A) Topographie du Massif des Grandes Rousses et localisation des bassins versants des lacs proglaciaires étudiés au Nord et au Sud du Massif. (B) Localisation des lacs et des sites archéologiques (M) étudiés au Nord et au Sud du massif, extensions actuelles des glaciers dans le massif (dont les glaciers instrumentés de Sarennes et de St Sorlin), et des cordons morainiques associés respectivement au PAG et aux stades du lac Blanc Huez, du lac Bramant et de l'Alpe d'Huez.
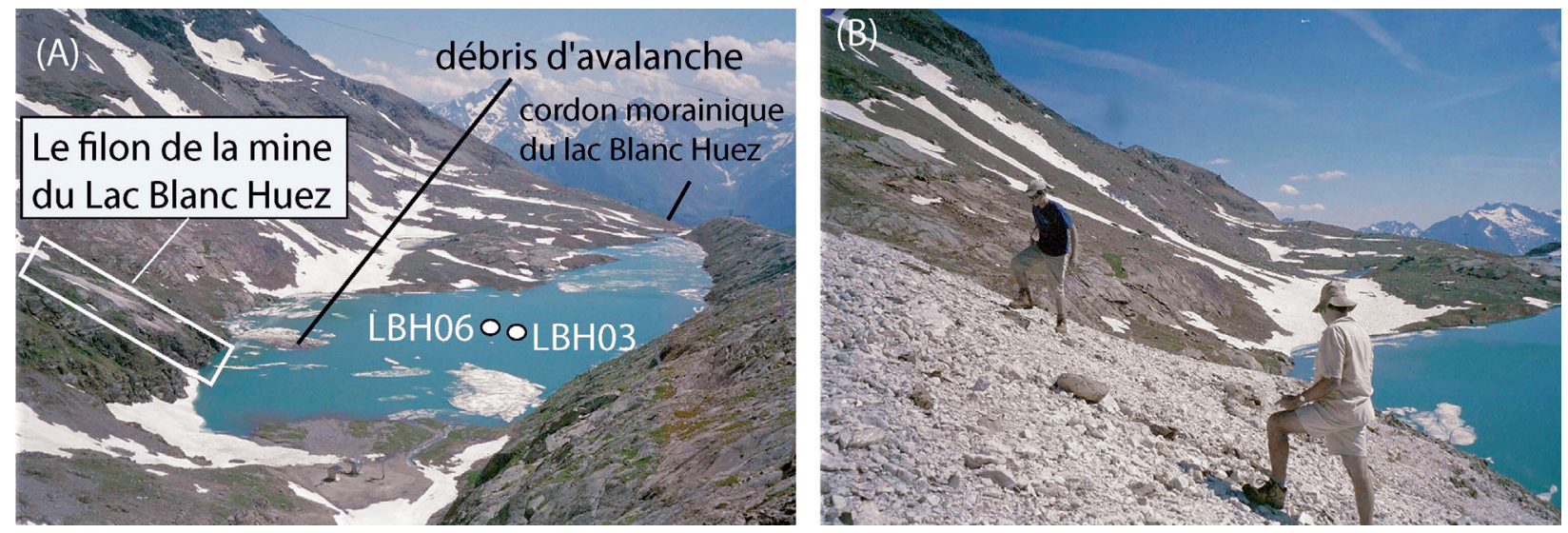

Figure 3 - Localisation de la mine médiévale du Lac Blanc Huez (filon et exhaure), des sites de carottages et du cordon morainique (A). Détail de l'exhaure composée de baryte et accumulé en bordure du filon (tranchée à ciel ouvert et réseau souterrain dans la partie amont du dépilage).

également développée entre AD 1236 et AD 1330 le long d'une faille minéralisée s'étendant sur plus de $120 \mathrm{~m}$ en bordure du Lac Blanc Huez (Figure 3). Plus récemment des chantiers d'extraction de chalcopyrite dans une gangue de quartz ont été identifiés au NW du massif entre 2200 et $2600 \mathrm{~m}$ d'altitude et datés du Bronze ancien (Bailly-Maitre et Gonon, 2006). Ces deux chantiers, détaillés ci-dessous, font encore actuellement l'objet d'études.

\section{II - MATÉRIElS ET MÉTHOdeS}

L'archéologie minière dans le massif des Grandes Rousses nécessite des recherches en altitude réalisées à ciel ouvert le long de filons, mais également des recherches souterraines par spéléologie dans les galeries. Ces recherches ont donc nécessité d'importants travaux de positionnements, de réouvertures, voir de désobstructions lorsque les galeries sont effondrées. L'accent est ici porté sur la nature des gisements, sur les modes d'exploitation de la roche et sur des fouilles systématiques au niveau des forges et des stériles reconnus. Les datations reposent essentiellement sur l'étude d'archives historiques, de datations radiocarbone et sur l'identification du mobilier céramique (Bailly-Maitre et Bruno-Dupraz, 1994).

Dans ce massif, seules les études géomorphologiques de Flusin et al. (1909) ont été effectuées avant le développement de la station de l'Alpe d'Huez initié dès les années 1930 et la réalisation en 1918 de barrages hydroélectriques ayant noyé les lacs de Bramant et de Blanc Bramant au Nord du massif (Guyard et al., 2007). Par la suite, les travaux de terrain plus détaillés de Monjuvent et Chardon (1989), Chardon (1991) et 
Edouard (1994) ont été réalisés avant que l'agrandissement de cette station de sports d'hiver ne perturbe profondément la géomorphologie et le drainage du versant SW de ce massif (Chapron et al., 2007). Ces résultats focalisés sur l'évolution des fronts glaciaires n'ont été calés chronologiquement que par les rares datations radiocarbone et palynologiques de Chardon (1991) effectuées au niveau de la station de l'Alpe d'Huez. Elles ont cependant grandement guidé le choix des lacs proglaciaires de Blanc Huez et de Bramant dont les remplissages sédimentaires ont fait l'objet d'une approche multidisciplinaire à très haute résolution.

Les approches limno-géologiques présentées en détail dans Chapron et al. (2007) et Guyard et al (2007) ont en effet englobé une cartographie acoustique par sismique réflexion à haute résolution (le système 3.5 $\mathrm{kHz}$ de l'ETH Zurich) et le prélèvement de carottages courts par gravité en Septembre 2003 (Figures 4 et 5). Ces données préliminaires positionnées par GPS ont permis d'optimiser le prélèvement un mois plus tard de deux carottages à piston au sein du remplissage sédimentaire le mieux préservé du Lac de Bramant (Figure 5) à partir du système à piston de l'Université de Bergen (Nesje, 1992). En Mars 2006, un carottage hivernal depuis la surface gelée du Lac Blanc Huez (mission TICO-TICO) à l'aide d'un carottier à piston de type UWITEC, nous a permis de prélever les $7 \mathrm{~m}$ qui composent le remplissage accumulé dans le bassin central de ce lac proglaciaire à proximité de la mine de plomb argentifère (Figures 3 et 4). Les propriétés physiques (gamma densimétrie, susceptibilité magnétique, micro-granulométrie laser, spectrophotométrie, tomographie scanner, radiographie numérique) et chimiques (teneur en matière organique, minéralogie, spectrométrie de masse ICP-MS, spectrométrie d'adsorption atomique et de fluorescence $\mathrm{X}$ sur banc ITRAX ) de ces sédiments lacustres (Figures 6 et 7) ont été établies dans les laboratoires de l'ETH Zurich, de l'Université

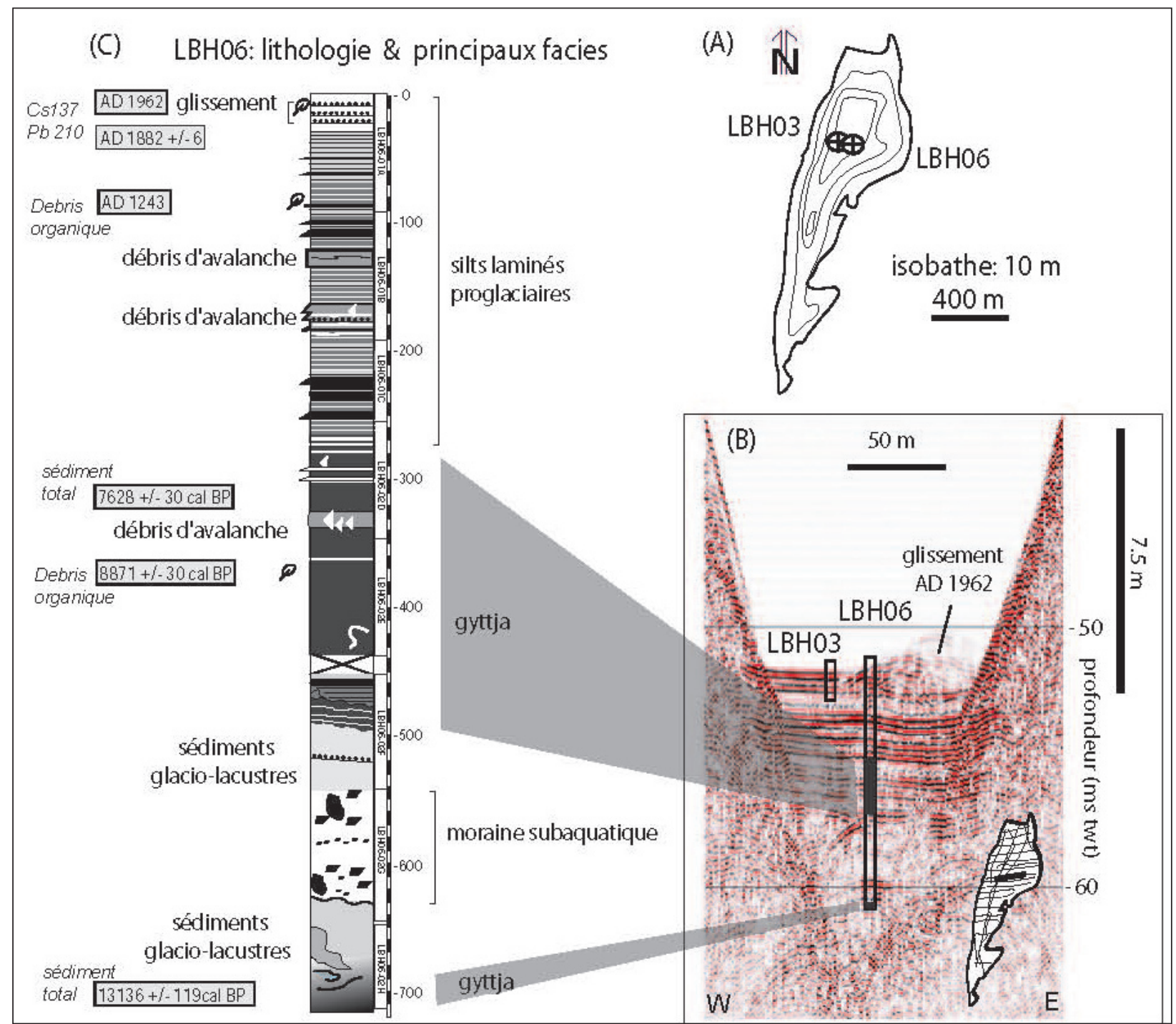

Figure 4 - Stratigraphie et chronologie du remplissage du Lac Blanc Huez établis à partir de carottages (A) localisés sur la base d'une cartographie "sismique réflexion » à haute résolution $(B)$ et de l'établissement d'un modèle âge-profondeur $(C)$ reposant sur des datations radionucléides et radiocarbone (effectuées sur macro-restes végétaux ou sédiment total). 


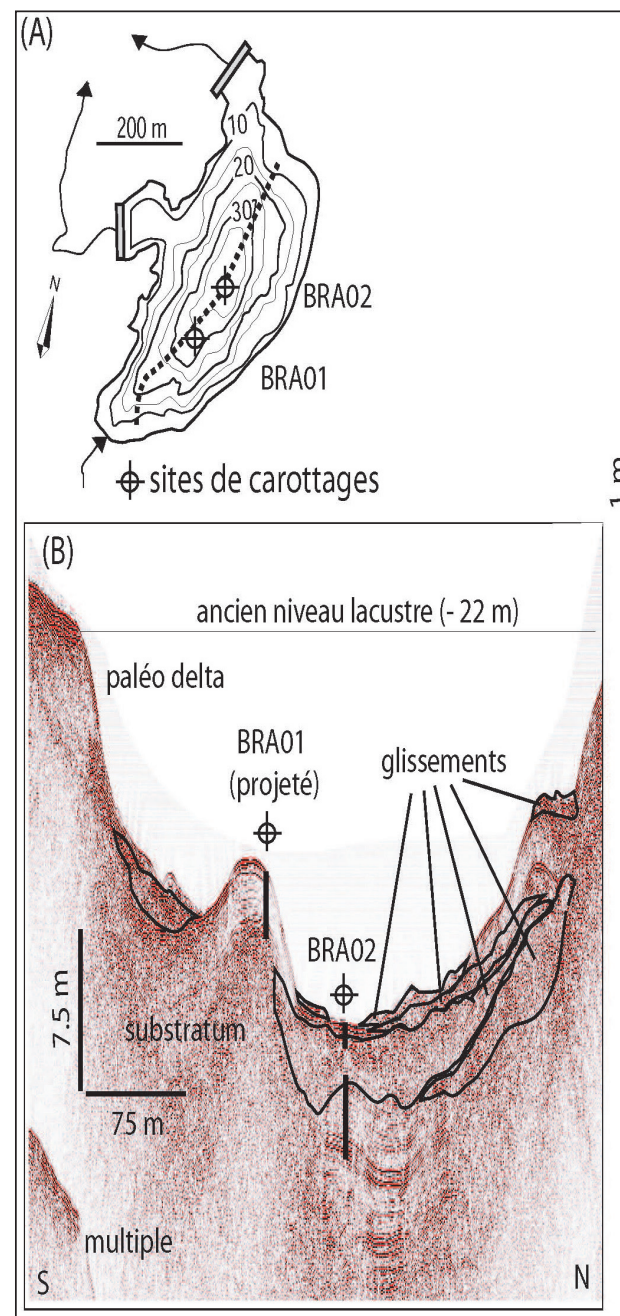

(C)

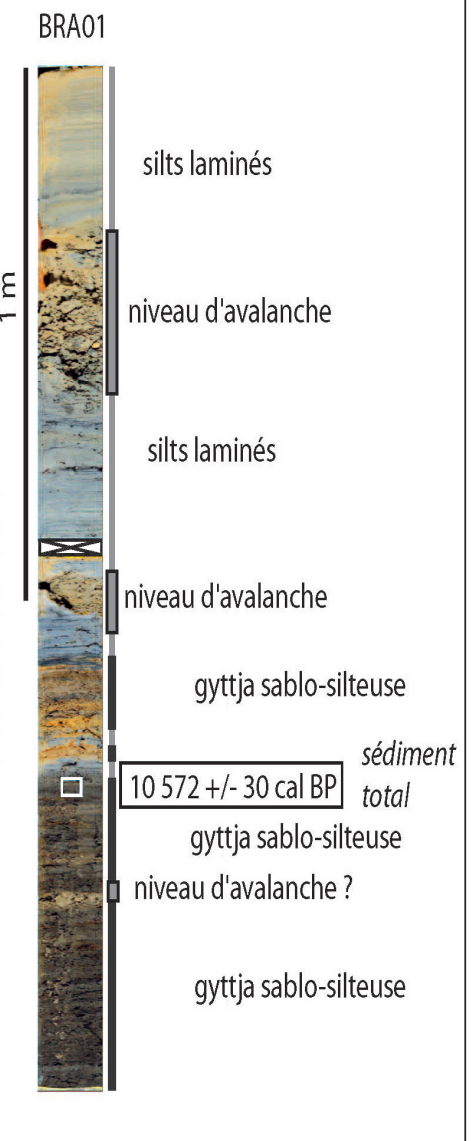

Figure 5 - Stratigraphie et âge minimum $d u$ remplissage du Lac de Bramant établis à partir de carottages (A) localisés sur la base d'une cartographie " sismique réflexion " à haute résolution $(B)$ et de l'établissement d'une datation radiocarbone effectuée sur sédiment total dans la carotte BRA01 (C).

Figure 6 - Identification par analyses ICP-MS de trois pics d'Argent dans la séquence néoglaciaire du Lac Blanc Huez. Les deux pics supérieurs sont reliés à l'exploitation de la mine de Plomb Argentifère du Lac Blanc Huez bien documentée par les archives entre AD 1236 et 1330 ; il est possible que le troisième pic corresponde soit à une première exploitation de ces minerais de baryte durant la période Romaine, soit durant l'Antiquité comme le suggère une des datations réalisées sur les dépôts accumulés dans le forge de la mine.

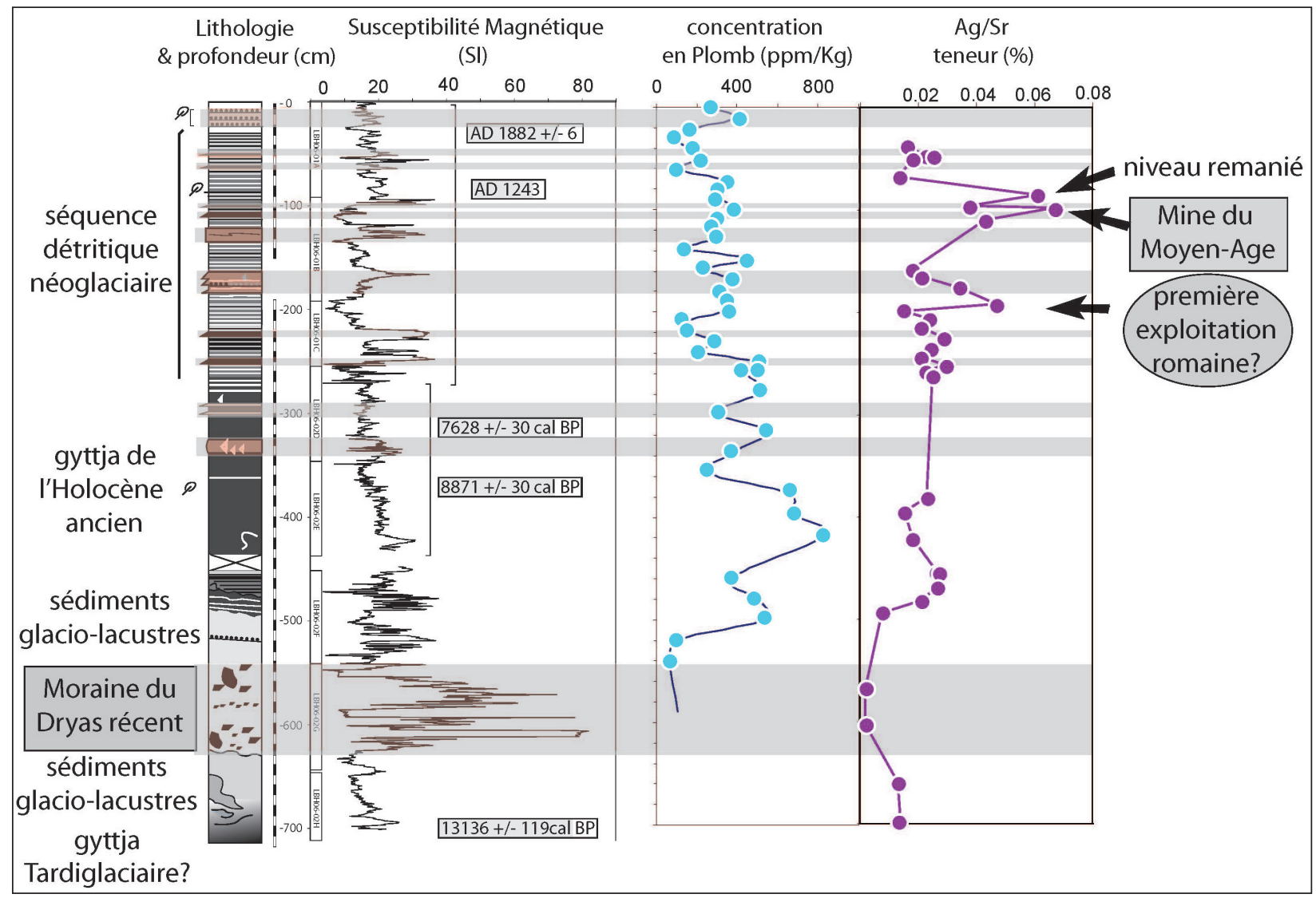




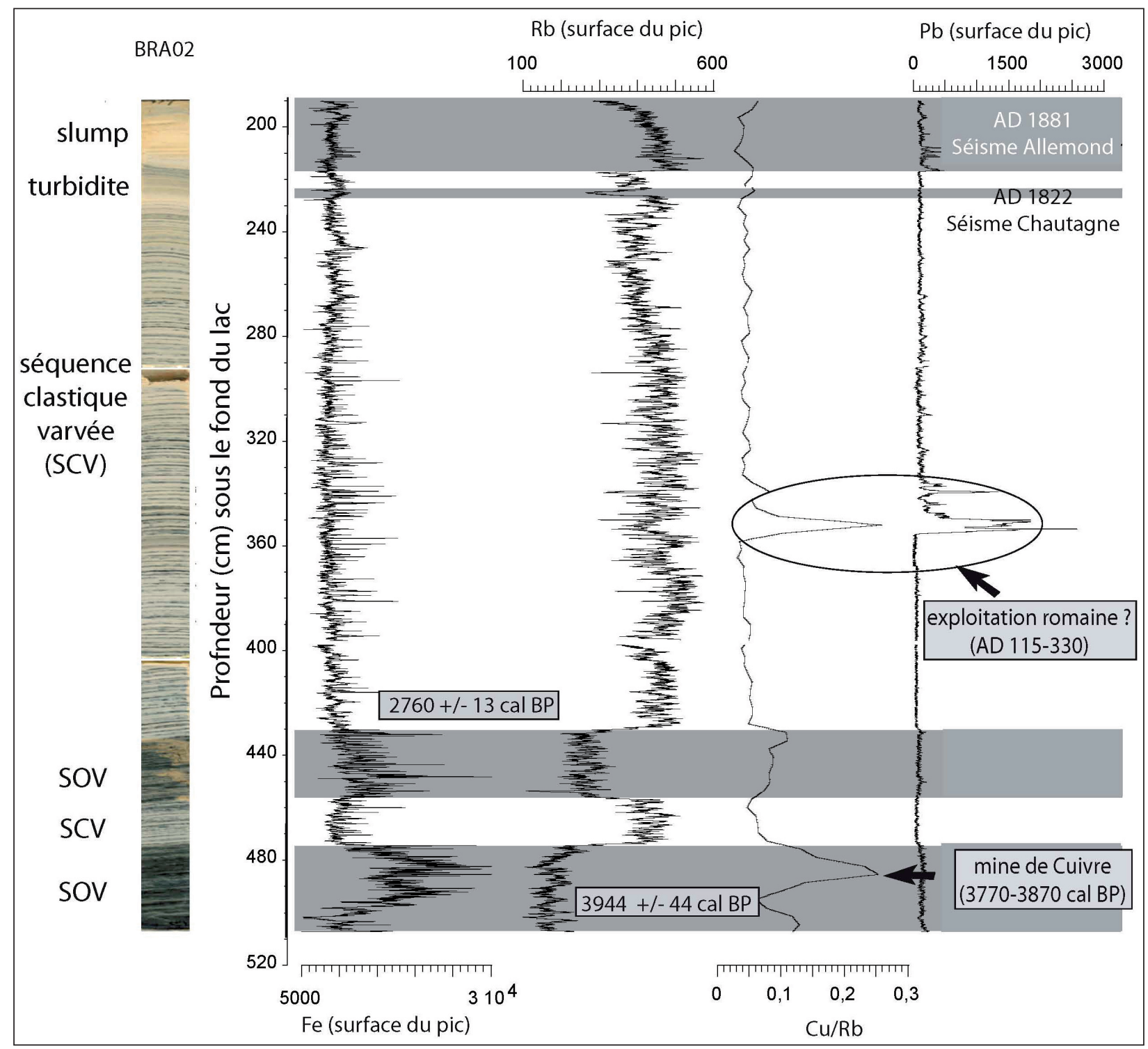

Figure 7 - Identification par fluorescence X d'un pic de Cuivre durant la période du Bronze ancien et d'un pic de Plomb couplé à un pic de Cuivre durant l'apogée de la période romaine dans les sédiments varvés du bassin central du Lac Bramant (carotte BRA02 située sous le slump de AD 1881, cf. Fig. 5). SOV : Séquence organique varvée. Modifié d'après Guyard et al. (2007).

de Savoie, de l'Observatoire de Grenoble, de l'ENTPE de Vaux en Velin, et du centre INRS-ETE de la ville de Québec. La chronologie des remplissages a été établie par datations radionucléides, radiocarbone AMS (sur macro-restes ou sur sédiment total) et grâce à la corré- lation de remaniements gravitaires subaquatiques avec les séismes historiques locaux et régionaux de $\mathrm{AD}$ 1962 (Corrençon), AD 1881 (Allemond) et AD 1822 (Chautagne) (Chapron et al., 1999 ; 2007 ; Nomade et al., 2005 ; Guyard et al., 2007).

\section{III - RÉSULTATS ET INTERPRÉTATIONS}

\section{1 - Les prospections archéologiques}

Les recherches menées depuis 1985 dans le massif ont permis d'identifier deux principaux ensembles miniers : les sites de Plan des Cavales 4 et de Barbarate 3.

Le site de Plan des Cavales 4 localisé sur un plateau incliné au NW du massif entre 2200 et 2600 m d'altitude (Figure 2), correspond à un site d'extraction de chalcopyrite dans une gangue de quartz. L'abattage au feu s'est fait à partir des affleurements. Plus de 40 indices de sites ont été cartographiés. Les datations radiocarbone obtenues sur les sites en falaise et en plateau situent l'exploitation au Bronze ancien (3800+/-100 cal BP ; 
Bailly-Maitre et Gonon, 2006).

Le second ensemble se trouve au NE du Lac Blanc Huez, à $2550 \mathrm{~m}$ d'altitude (site de Barbarate 3). Il s'agit d'une exploitation de plomb argentifère dans une gangue de baryte le long de la falaise constituant le flanc Est du lac (Figure 3). La chronologie des travaux miniers est incertaine ou large. Trois datations ont été obtenues avec des méthodes différentes : (i) un âge radiocarbone AD 1070-1285 obtenu sur un bois pris au pied d'une paroi de galerie, (ii) un âge radiocarbone 350 cal BC-AD 20 mesuré sur des charbons de bois provenant de la forge associée à la mine, et (iii) une datation des XII-XIV ${ }^{\mathrm{e}}$ siècles après notre ère déduite du mobilier céramique provenant de la forge.

Il semble donc possible que le filon du Lac Blanc Huez ait été exploité durant l'Antiquité mais cela reste à prouver par l'archéologie.

\section{2 - La sédimentation lacustre proglaciaire}

Les recherches pluridisciplinaires réalisées depuis 2003 dans les lacs Blanc Huez et Bramant permettent de préciser la nature de ces remplissages et la chronologie de fluctuations glaciaires dans leur bassin versant : celles des glaciers des Rousses et de Herpie au SE du massif dans le bassin du Lac Blanc Huez, et celles du glacier de St Sorlin au Nord du massif dans le bassin du Lac de Bramant.

Le remplissage du Lac Blanc Huez se caractérise dans le sondage LBH06 long de $7 \mathrm{~m}$ (Figure 4) par un fin niveau de gyttja daté de 13136 +/- 119 cal BP, surmonté par une séquence glacio-lacustre de couleur gris foncé, encadrant un dépôt métrique de moraine subaquatique gris clair contenant de nombreux débris rocheux pluri-centimétriques anguleux dans une fine matrice fortement compactée. Cette séquence glacio-lacustre évolue au-dessus d'un contact érosif en une séquence pluri-métrique de gyttja de couleur noire datée de l'Holocène ancien dans laquelle s'intercale un niveau plus grossier riche en petits débris rocheux anguleux. Sur la base des observations de terrains réalisées durant l'été 2005 (Figure 3), ce niveau est interprété comme étant le produit de la décantation des débris accumulés sur la surface gelée du lac à l'occasion d'une avalanche de neige érosive, lors de la fonte des icebergs qui se forment et disparaissent chaque année (Chapron et al., 2007). La séquence de gyttja évolue progressivement vers $250 \mathrm{~cm}$ de profondeur sous le niveau du lac en une séquence pluri-métrique de silts finement laminés (alternance de lamines grises et beiges) et fréquemment interrompus par des niveaux de débris d'avalanche et des niveaux sablo-silteux granoclassés centimétriques, typiques de niveaux de crues (Chapron et al., 2007). Dans le premier mètre sous la surface du lac, un niveau granoclassé contenant des débris organiques, interprété comme étant un dépôt de crue, a donné un âge AD1243+/-19, et un glissement gravitaire pluri-centi- métrique, situé sous l'interface eau-sédiment, est daté par datation radionucléides et par corrélation avec la carotte LBH03 de AD 1962. Ce remaniement gravitaire a donc été très probablement produit par la propagation des ondes sismiques lors du tremblement de terre de Corrençon en 1962.

L'évolution de la susceptibilité magnétique (SM), des concentrations en Plomb total et du rapport $\mathrm{Ag} / \mathrm{Sr}$, mesurés selon différents pas d'échantillonnages sur la carotte LBH06, est présentée dans la figure 6. La synthèse de ces analyses, de la lithologie et la chronologie du remplissage suggèrent que ce lac a été déglacé durant le Tardiglaciaire (formation d'une gyttja dans un petit lac de type organique durant l'Allerød probablement), puis partiellement recouvert par la recrudescence des glaciers des Rousses et d'Herpie dans un environnement glacio-lacustre proximal (langue glaciaire débouchant sur un lac juxta-glaciaire) dont le développement maximal (probablement durant le refroidissement du Dryas Récent entre 12890 et 11650 cal BP) a induit le dépôt d'une langue morainique (till tongue). Le retrait des glaciers en contexte glacio-lacustre au début de l'Holocène a probablement induit des changements abrupts de la cote du lac, comme le suggèrent les contacts érosifs identifiés au sommet de la séquence glacio-lacustre supérieure et à la base de l'unité de gyttja Holocène. La mise en place de cette unité organique durant l'Holocène ancien suggère une disparition quasi totale des glaciers dans le bassin versant du lac durant plusieurs millénaires. L'accumulation de débris d'avalanche indique cependant que le lac a pu être gelé durant certains hivers et que d'importantes précipitations neigeuses ont pu se produire. Le développement d'un néoglaciaire durant l'Holocène moyen serait ici à l'origine de la transition progressive d'une sédimentation organique de type gyttja, vers une sédimentation largement plus détritique (accumulation de farine glaciaire) et finement laminée (formation de varves glaciaires ?). De plus fréquents dépôts d'avalanches et de nombreux niveaux de crue suggèrent également ici la recrudescence des précipitations sous forme de neige favorisant le développement des glaciers et une intensification des crues de printemps résultant de la fonte des neiges et des glaces. La fluctuation de la SM des sédiments suggère également que la taille des glaciers dans le bassin versant a varié de façon significative durant ce néoglaciaire, avant d'atteindre un développement maximal durant le PAG (Chapron et al., 2007).

Le remplissage du Lac Blanc Huez se traduit cependant par des teneurs en Plomb remarquablement fortes ( $>800 \mathrm{ppm}$ au début de l'Holocène) et variables (Figure 6). De telles concentrations au sein de sédiments organiques suggèrent ici l'importance de phénomènes d'adsorption des métaux par les particules organiques (dans les sols du bassin versant ou dans le remplissage lacustre) et limite donc l'utilisation du signal Plomb comme indicateur des activités minières 
à proximité du lac. Il est également ici probable qu'une partie de la variabilité du signal de SM soit due à la présence de métaux dans les sédiments, ce qui limite l'utilisation de ce marqueur comme indicateur paléoclimatique.

Trois niveaux particulièrement riches en $\mathrm{Ag}$ à $190 \mathrm{~cm}, 100 \mathrm{~cm}$ et $90 \mathrm{~cm}$ de profondeur sont cependant clairement supérieurs au bruit fond géochimique de cet élément (Figure 6), dont les teneurs restent normalement très faibles dans les sédiments quaternaires. Ces trois pics suggèrent donc l'impact d'exploitations anciennes des filons de Plomb Argentifère qui ont été documentées à proximité du lac et de son bassin versant (cf. Bailly-Maitre et Bruno-Dupraz, 1994). Le pic à $90 \mathrm{~cm}$ correspond en effet à un niveau de crue remarquablement riche en débris organiques dont l'âge (AD $1243+/$ - 19) suggère que le carbone résulte de l'exploitation de la mine du Lac Blanc Huez durant le Moyen Age (abattage au feu, ou aménagement de galeries). Le pic à $100 \mathrm{~cm}$ semble donc également être ici lié à l'exploitation de la mine durant le Moyen Age. Les registres historiques indiquant clairement que cette exploitation d'altitude entre AD 1236 et 1330, a du être achevée en raison de graves problèmes d'inondations des galeries, il semble que le niveau riche en $\mathrm{Ag}$ à $100 \mathrm{~cm}$ marque ici le début des exploitations et que le niveau de crue à $90 \mathrm{~cm}$ résulte plutôt d'une contamination induite par le lessivage des galeries durant le XIVème siècle. Le pic à $190 \mathrm{~cm}$ semble lui résulter d'une première phase d'exploitation du Plomb Argentifère plusieurs siècles auparavant (Antiquité ou période romaine ?).

Dans le Lac de Bramant, deux carottages ont été prélevés sur la base des explorations sismiques (Figure 5) afin (i) de préciser l'âge de la formation de ce bassin lacustre à la faveur du retrait du glacier de St Sorlin (site BRA01, Figure 5) et (ii) de documenter la nature du remplissage dans le bassin principal et l'évolution des conditions paléo-environnementales (site BRA02, Figures 5 et 7).

La carotte BRA01 longue de $2 \mathrm{~m}$, a été prélevée au sein d'une séquence sédimentaire drapant un épaulement du substratum. Elle se compose à la base d'une séquence de gyttja sablo-silteuse de couleur brune dont le sommet a pu être daté de $10572+/-30$ cal BP. Cette première séquence est coiffée par $8 \mathrm{~cm}$ de silts laminés de couleur grise, puis une nouvelle séquence de gyttja sablo-silteuse de couleur brune épaisse de $12 \mathrm{~cm}$, ellemême recouverte par une séquence silteuse de couleur grise, clairement laminée et interrompue par deux événements érosifs correspondant à des niveaux d'avalanches ou de coulées de boues (debris flows). Ces niveaux contiennent de nombreux débris rocheux anguleux parfois pluri-centimétriques (Figure 5). Le sommet du niveau d'avalanche supérieur est enfin coiffé par une séquence de silts laminés de couleur brun clair.

Ce sondage permet donc d'attribuer un âge minimum au Lac de Bramant remontant à la période du Préboréal. Ce bassin lacustre initialement organique et probablement peu profond (les gyttjas sablo-silteuses sont généralement littorales (Veres et al., 2007), a par la suite évolué en un système clastique favorisant la formation d'un delta (aujourd'hui ennoyé sous $22 \mathrm{~m}$ de profondeur par le barrage hydroélectrique de AD 1918 (Figure 5). La mise en place de deux dépôts d'avalanches érosives suggère d'importantes précipitations hivernales durant l'Holocène et la présence d'importants hiatus dans cette carotte.

La carotte BRA02, longue de $3 \mathrm{~m}$, prélevée entre 2 et $5 \mathrm{~m}$ sous le fond du lac à la base d'une série de glissements gravitaires historiques identifiés sur les profils sismiques et recouvrant tout le bassin central (Guyard et al., 2007 ; Figure 5), est finement laminée dans son ensemble et caractérisée à la base par l'alternance de deux séquences organiques intercalées entre deux séquences clastiques (Figure 7). Le décompte à partir des données XRF des lamines noires (enrichies en Fer, en matière organique et de granulométrie plus grossière) et des lamines claires (enrichies en Rubidium et de granulométrie plus fine), ainsi que la détermination de l'âge de deux macro-restes végétaux (3944 +/- 44 cal BP dans la séquence organique inférieure et $2760+/-13$ cal BP à la base de la séquence clastique supérieure) a révélé la formation d'une lamination annuelle (formation de varves glaciaires). L'existence de varves dans l'ensemble de cette carotte est également confirmée par la corrélation de deux événements gravitaires (une turbidite et un slump) avec le séisme historique régional de Chautagne et le séisme local d'Allemond (Chapron et al., 1999 ; Nomade et al., 2005 ; Guyard et al., 2007). Cette chronologie détaillée et la mise en place d'une sédimentation proglaciaire essentiellement organique (jusqu'à $4 \%$ de Carbone Organique Total) durant le Bronze ancien (4250-3600 cal BP) et le Bronze récent (3300-2700 cal BP), uniquement dans la partie profonde du bassin (la répartition des deux réflecteurs de forte amplitude à la base du carottage dans la Fig. 5), implique une sédimentation de type anoxique (présence de souffre dans les sédiments) dans un lac peu profond déconnecté des apports hydriques et particulaires provenant de la diffluence du glacier de St Sorlin. Inversement, les séquences clastiques proglaciaires durant le Bronze moyen (3600-3300 cal BP) et depuis le début de l'Age du Fer, ainsi que la répartition des dépôts depuis 2800 ans cal BP (formation d'un delta et sédimentation couvrant une plus large surface $\mathrm{du}$ bassin) résulte d'une sédimentation dans un lac beaucoup plus vaste et plus profond, largement soumis aux apports hydriques et particulaires du glacier de St Sorlin (Guyard et al., 2007).

Ce sondage indique donc clairement que le bassin versant du Lac de Bramant a subi d'importants changements environnementaux durant l'Age du Bronze : deux périodes de bas niveaux lacustres déconnectées des apports du glacier de St Sorlin (durant le Bronze ancien et le Bronze récent) ; une brève transgression lacustre durant l'Age du Bronze moyen et la mise en 
place d'une transgression depuis l'Age du Fer, induites toutes les deux par la diffluence du glacier de St Sorlin dans la vallée des Bramants.

Les données XRF sur ce sondage illustrent également l'existence d'un premier pic de Cuivre entre 3770 et 3870 cal BP, puis d'un second pic de Cuivre couplé à un très net pic de Plomb entre AD 115 et 330 (Figure 7). Le premier pic de Cuivre durant le Bronze ancien peut être relié aux récentes découvertes des sites d'extraction à ciel ouvert de la chalcopyrite sur le site de Plan des Cavales 4 à proximité du bassin versant du Lac de Bramant ; les pics de Cuivre et de Plomb durant l'apogée de la période Romaine pourraient eux résulter (i) soit d'une phase d'extraction de minerais dans le massif des Grandes Rousses, (ii) soit d'une pollution atmosphérique plus régionale résultant d'extractions plus lointaines (Guyard et al., 2007).

\section{IV - Discussion}

Les nouvelles données chrono-stratigraphiques détaillées ci-dessus suggèrent que les lacs Blanc Huez et Bramant ont été déglacés depuis la fin du Tardiglaciaire et que leurs bassins versants ont été très faiblement englacés durant la première partie de l'Holocène. La seconde partie de notre interglaciaire se caractérise par la mise place d'une période néoglaciaire favorisant le retour d'une sédimentation détritique (parfois varvée) dans les lacs proglaciaires. L'activité glaciaire semble cependant avoir été variable durant ce néoglaciaire, comme par exemple durant l'Age du Bronze ancien et récent, lorsque les glaciers étaient probablement plus réduits qu'ils ne le sont aujourd'hui. L'extension maximale des glaciers holocènes aurait enfin été atteinte récemment, durant le PAG.

La remontée de la ligne d'équilibre des glaciers durant l'Holocène ancien semble avoir provoqué des fluctuations lacustres et favorisé une sédimentation plus organique dans les bassins lacustres proglaciaires. Le retrait des glaciers semble également avoir permis une remontée en altitude de la limite supérieure de la forêt, puisque plusieurs macro-restes végétaux datés de $10570,8870,3900$ et 2800 ans cal BP environ, ont été retrouvés dans les séquences lacustres à plus de $2500 \mathrm{~m}$ d'altitude.

Le pic du Cuivre identifié dans le remplissage du Lac de Bramant durant la période du Bronze ancien entre 3770 et 3870 ans cal BP constitue, à ce jour, l'enregistrement sédimentaire non seulement le plus élevé en altitude, mais également le plus ancien, de retombées atmosphériques induites par le développement de la paléo-métallurgie sur le territoire français.

Une période de retrait glaciaire au nord du massif des Grandes Rousses durant le Bronze ancien semble avoir permis des conditions environnementales favorables à l'exploration et l'exploitation de gisements métallifères rendus plus accessibles (après la fonte des neiges). Il est également possible que la remontée de la limite supérieure de la forêt au-delà de $2500 \mathrm{~m}$ d'altitude, ait facilité l'extraction du minerai par abattage au feu.

L'identification de deux pics d'argent dans les sédiments du Lac Blanc Huez à 90 et $100 \mathrm{~cm}$ sous la surface du lac dont les valeurs sont bien au-delà du bruit de fond géochimique (Figure 6), et l'âge du pic supérieur mesuré sur un niveau remanié (AD 1243+/-19), per- mettent de relier ces deux pics à l'impact local de l'exploitation de la mine de plomb argentifère du Lac Blanc Huez durant le Moyen Age entre AD 1236 et 1330. Sur la base des archives écrites de la mine, le premier pic d'Ag identifié à $100 \mathrm{~cm}$ dans la carotte LBH06 serait ainsi probablement lié à l'ouverture de cette exploitation en $\mathrm{AD}$ 1236. L'origine du second pic identifié à $90 \mathrm{~cm}$ et daté de l'an 1243 pourrait par contre plutôt résulter de phénomènes de lessivage des galeries dont l'ennoiement de plus en plus fréquent a entraîné l'arrêt de la mine en AD 1330.

Il semble donc ici que l'arrêt de l'exploitation de cette mine à $2500 \mathrm{~m}$ d'altitude aurait résulté du changement rapide des conditions climatiques associées à l'initiation du Petit Age Glaciaire au Moyen Age.

La reconnaissance d'un pic de Plomb et de Cuivre dans le lac de Bramant durant l'apogée de la période romaine, et d'un pic d'Argent dans le remplissage du Lac Blanc Huez à $190 \mathrm{~cm}$ sous la surface, est par contre encore difficilement explicable (Figures 6 et 7).

En effet bien que la présence de Cuivre dans le lac de Bramant et celle d'Argent dans le lac Blanc Huez suggèrent l'enregistrement d'une activité minière locale, sur la base des données chronologiques disponibles dans ces deux lacs, il n'est pas encore possible d'affirmer que ces pics de métaux identifiés dans les remplissages lacustres proglaciaires, aux deux extrémités du massif des Grandes Rousses, sont contemporains. A ce jour, aucun indice archéologique ne permet non plus d'établir le développement d'activités métallurgiques durant la période romaine dans ce massif. Les pics de métaux du Lac de Bramant pourraient donc résulter d'exploitations romaines plus lointaines, d'origine alpine ou européenne (Boutron et al., 2004 ; Arnaud et al., 2005 ; Durali-Mueller et al., 2007). Dans ce cas, le remarquable pic d'argent à $190 \mathrm{~cm}$ de profondeur dans le Lac Blanc Huez pourrait résulter d'une exploitation locale durant l'Antiquité, comme le suggère la datation de charbons de bois provenant de la forge associée à la mine du lac Blanc Huez.

$\mathrm{Si}$ les recherches en cours permettent de confirmer la contemporanéité des pics de métaux identifiés dans les remplissages des lacs Bramant et Blanc Huez, cela pourrait traduire l'enregistrement sédimentaire d'une première phase d'exploration et d'exploitation des 
minerais généralisée dans l'ensemble du massif des Grandes Rousses durant la période romaine. Il est en effet possible que la préservation de sites d'exploitations minières de la période romaine dans ce massif ait été fortement altérée par le développement de l'activité glaciaire durant le PAG. Cette hypothèse implique- rait que le déboisement intensif observé par Chardon (1991) sur le plateau de l'Alpe d'Huez à $2000 \mathrm{~m}$ d'altitude durant la période romaine, aurait pu résulter, à la fois du développement de la paleo-métallurgie dans le massif, et du pastoralisme sur les plateaux accessibles dans ce même massif.

\section{V - Conclusions et Perspectives}

Les nouvelles données chrono-stratigraphiques établies dans les remplissages des lacs de Bramant et de Blanc Huez à partir de cartographies sismiques, de datations radiocarbone, de données historiques et de l'identification de pics de pollution atmosphériques, illustrent la richesse et la sensibilité de ces archives naturelles aux changements de l'environnement d'origine climatique (les fluctuations glaciaires) depuis la fin du Tardiglaciaire, et aux développements des activités métallurgiques d'altitude dès la période du Bronze ancien entre 3700 et 3800 ans cal BP.

Ces recherches indiquent que l'exploitation ancienne de filons métallifères dans ce massif peut être documentée à partir de la mesure des teneurs en Cuivre et en Plomb des sédiments dans le Nord du massif, et à partir des teneurs en Argent des sédiments au Sud du massif.

Afin de préciser la chronologie des fluctuations glaciaires durant l'Holocène, ainsi que l'origine et l'évolution des exploitations de Plomb durant la période romaine, les recherches en cours consistent donc à identifier : (i) l'origine de la matière organique accumulée dans les sédiments lacustres avant de réaliser de nouvelles datations radiocarbone sur sédiment total permettant d'affiner les modèles âge-profondeur et notamment de préciser l'âge du néoglaciaire dans ce massif, et (ii) la signature isotopique des contaminations au plomb afin de préciser l'existence de sources locales ou plus lointaines.

\section{Remerciements}

Nous tenons à sincèrement remercier l'Institut de la Montagne et le GDR Juralp pour leur soutien financier et adressons de chaleureux remerciements à Xavier Fain, David Goutaland et Maxime Debret pour leur aide précieuse sur le terrain lors de la campagne TICO-TICO. Ces études de terrain ont également grandement été facilitées sur le lac de Bramant par l'aide de la commune de St Sorlin d'Arve et de Bruno Axelard (refuge CAF) et sur le lac Blanc Huez par le soutien logistique de la SATA de l'Alpe d'Huez.

\section{BibLIOGRAPHIE}

Alfonso S, Goursset F, Massé L., Tastet J.-P., 2001. A European lead isotope signal recorded from 6000 to 300 years BP in coastal marshes (SW france). Atmospheric Environment, 35, 3595-3605.

Arnaud F, Serralongue J, Winiarski T, Desmet M, Patern M, 2005. Pollution au plomb dans la Savoie antique (II-III $\mathrm{e}$ s. ap. J-C) en relation avec une installation, métallurgique de la cité de Vienne. C.R. Geoscience, 338, 244-252.

Bailly-Maitre M.-C., Bruno-Dupraz J., 1994. Brandes en Oisans: La mine d'argent des Dauphins (XII-XIVe siècles) Isère. Documents d'Archéologie en Rhône-Alpes, vol. 9. 172 pp.

Bailly-Maitre M.C., Gonon T., 2006. L'exploitation de la chalcopyrite à l'Age du Bronze dans le massif des Rousses (Oisans-Isère). In Proceedings of the 131 st Congress of the CTHS. "Tradition et innovation," Grenoble, avril 2006.

Boutron C, Rosman K, Barbante C, Bolshov M, Adams S, Hong F, Ferrari C, 2004. L'archivage des activités humaines par les neiges et glaces polaires: le cas du plomb, CR. Geoscience, 336, 847-867.
Chapron E., Desmet M., De Putter T., Loutre M.F., Beck C., Deconinck J.F., 2002. Climatic variability in the northwestern Alps, France, as evidenced by 600 years of terrigenous sedimentation in Lake Le Bourget. The Holocene, 12 (2), 177-185.

Chapron E, Fain X, Magand O, Charlet L, Debret M, MÉLIĖRes M.-A., 2007. Reconstructing recent environmental changes from proglacial lake sediments in the Western Alps (Lake Blanc Huez, 2543 m a.s.l., Grandes Rousses Massif, France). Palaeogeography, Palaeoclimatology, Palaeoecology, 252, 586-600.

Chardon M., 1991. L'évolution tardiglaciaire et holocène des glaciers et de la végétation autour de l'Alpe d'Huez (Oisans, Alpes Françaises). Revue de Géographie Alpine, 2, 39-53.

Deline, P., Orombelli, G., 2005. Glacier fluctuations in the western Alps during the Neoglacial, as indicated by the Miage morainic amphitheatre (Mont Blanc massif, Italy). Boreas, 34, 456-467.

Dorthe-Monachon C, 1988. Les variations glaciaires dans la vallée de l'Arve (Haute Savoie) après le dernier maximum wurmien et jusqu'à l'Holocène. Quaternaire, $2 / 3,97-101$ 
Durali-Mueller S, Grey GP, WigG-Wolf D, Lahaye Y, 2007. Roman lead mining in Germany : its origin and development through time deuced from lead isotope provenance studies. Journal of Archeological Science, 34, $1555-1567$.

Edouard J.-L., 1994. Les lacs d'altitude dans les Alpes françaises. Contribution à la connaissance des lacs d'altitude et à l'histoire des milieux montagnards depuis la fin du Tardiglaciaire. HDR, Université de Grenoble, $790 \mathrm{p}$.

Flusin G., JacoB C., Offner J., 1909. Etudes glaciaires, géographiques et botaniques dans le massif des Grandes Rousses. Etudes glaciologiques. Ministère de l'Agriculture, Paris, 107-112.

Galop D, Tual M, Monna F, Dominio J, Beyrie A, Marembert $\mathrm{F}, 2001$. Cinq millénaires de métallurgie en montagne basque. Les apports d'une démarche intégrée alliant palynologie et géochimie isotopique du plomb. Sud-Ouest Européen, 11, 3-15.

Guyard H, Chapron E, St Onge G, Anselmetti FS, Arand F, Magand O, Francus P, Mélières M.-A., 2007. Highaltitude varve records of abrupt environmental changes and mining activity over the last 4000 years in the Western French Alps (Lake Bramant, Grandes Rousses Massif). Quaternary Science Reviews, doi : 10.1016/j.quascirev. 200707.007.

Holzhauser H., Magny M. and ZumbÜHL H., 2005. Glacier and lake-level variations in west-central Europe over the last 3500 years. The Holocene, 15, 6, 789-801.

Hormes A, Muller B, Schluchter C, 2001. The Alps with little ice: evidence for eight Holocene phases of reduced glacier extent in the Central Swiss Alps. The Holocene, $11,255-265$

Jouffroy-Bapicot I, Pulido M, Baron S, Galop D, Monna F, Lavoie M, Ploquin A, Petit C, de Beaulieu Jl, Richard H, 2007. Environmental impact of early palaeometallurgy : pollen and geochemical analysis. Vegt. Hist. Archaeobot, $16,251-258$.

Leeman A., Niessen F., 1994. Holocene glacial activity and climatic variations in the Swiss Alps: reconstructing a continuous record from proglacial lake sediments. The Holocene, 4, 259-268.
Magny M., HaAs J.N., 2004. A major widespread climatic change around $5300 \mathrm{cal}$. Yr BP at the time of the Alpine Iceman. Journal of Quaternary Science, 19, 1-8.

Monna F, Galop D, Carozza L, Tual M, Beyrie A, Marembert F, Chateau C, Dominik J, Grousset F, 2004. Environmental impact of early Basque mining and smelting recorded in a high ash minerogenic peat deposit. Science of the Total Environment, 327, 197-214.

Monjuvent, G., Chardon, M., 1989. Les extensions glaciaires des versants Ouest et Sud des Grandes Rousses. Internal report from Institut Dolomieu and Institut de Géographie Alpine. CNRS/University of Grenoble. 32 pp.

Nesje A., 1992. A piston corer for lacustrine and marine sediments. Arctic and Alpine Research, 24, 257-259.

Nesje, A., Lie, O., Dhal, S.O., 2000. Is the North Atlantic Oscillation reflected in Scandinavian glacier mass balance records? Journal of Quaternary Science, 15, 587-601.

Nomade J., Chapron E., Desmet M., Reyss J-L., Arnaud, F., LIGNER V., 2005. Reconstructing historical seismicit from lake sediments (Lake Laffrey, Western Alps, France). Terra Nova, 17, 350-357.

Rosman K, Chisholm W, Hong S, Candelone J.-P., Boutron C, 1997. Lead from Carthaginian and Roman Spanish mines isoptopically identified in Greenland ice dated from 600 BC tp 300 AD. Environ. Sci. Technol., 31, 3413-3416.

Solomina O, Haeberli W, Kull C, Wiles G. (2007). Historical and Holocene glacier-climate variations: general concepts and overview. Global and Planetary Change.

Torinesi O., Letreguilly A., Valla F., 2002. A century reconstruction of mass-balance of Glacier de Sarennes, French Alps. Journal of Glaciology, 48, 142-148.

VINCENT C., 2002. Influence of climate change over the 20th Century on four French glacier mass balances. Journal of Geophysical Research, 107.

Vincent C., Le Meur E., Six D., 2005. Solving the paradox of the end of the Little Ice Age in the Alps. Geophysical Research Letters, 32. 